\title{
Desenvolvimento de Superfícies de Energia Potencial para Sistemas de Cinco Corpos com Caráter Quiral
}

Patrícia R. P. Barreto, Alessandra F. Albernaz, Glauciete S. Maciel, Federico Palazzetti, Andrea Lombardi \& Gaia Grossi

Este trabalho é calculada o potencial de interação entre $\mathrm{o}_{2} \mathrm{O}_{2}$ e $\mathrm{H}_{2} \mathrm{~S}_{2}$ com os gases nobres, $\mathrm{He}, \mathrm{Ne}, \mathrm{Ar}, \mathrm{Kr}$, e Xe. O peróxido e persulfeto de hidrogênio foram modelados como moléculas rígidas, exceto pelo modo torsional ao londo da ligação $\mathrm{X}-\mathrm{X}$, com $\mathrm{X}=$ O ou S. A maior preocupação foi a definição do sistema de coordenadas e a expansão nos harmônicos hiperesféricos para o potencial, para que o mesmo reproduzisse as propriedades geométricas e de simetria dos sistemas, tornando-o um protótipo para a interação entre um átomo e uma molécula fracamente ligada.

Palavras-chave: superficie de energia potencial; sistemas quirais; interação de van der Waals.

A quantum chemical exploration is reported on the interaction potentials of $\mathrm{H}_{2} \mathrm{O}_{2}$ and $\mathrm{H}_{2} \mathrm{~S}_{2}$ with the rare gases, $\mathrm{He}, \mathrm{Ne}, \mathrm{Ar}, \mathrm{Kr}$, and $\mathrm{Xe}$. Hydrogen peroxide and persulfite is modeled as rigid except for the torsional mode around the $\mathrm{X}-\mathrm{X}$ bond, with $\mathrm{X}=\mathrm{O}$ or $\mathrm{S}$. Particular attention is devoted to the definition of coordinates and expansion formulas for the potentials, allowing for a faithful representation of geometrical and symmetry properties of these systems, prototypical of the interaction of an atom with a floppy molecule.

Keywords: potential energy surface; chiral system; van der Waals interactions. 


\section{Introdução}

Em química da atmosfera, recentemente a atenção tem sido direcionada para os fenômenos intermoleculares responsáveis pela formação de dímeros ou até clusters maiores que conduzem a efeitos mensuráveis de absorção de radiação. Além dos componentes principais da atmosfera, $\mathrm{O}_{2}$ e $\mathrm{N}_{2}$, outras moléculas, por exemplo, a água, também tem um papel importante. $\mathrm{O}$ objeto deste artigo está relacionado com o tratamento quântico da dinâmica de clusters para a caracterização de efeitos específicos e que podem ser medidos experimentalmente por espalhamento por feixe molecular para a determinação das interações intermoleculares.

A interação fraca de moléculas que conduzem a complexos colisionais complexos, estáveis ou dímeros metaestáveis, tem um papel importante na física de superfícies, astrofísica, fotoquímica e física da atmosfera e mudanças climáticas. Espectros de alta resolução de clusters de van der Waals podem fornecer informações sobre a natureza das forças intermoleculares e a dinâmica interna, porém os resultados para moléculas não polares são limitados.

\section{SUPERFÍCIE DE ENERGIA POTENCIAL: $\mathrm{O}_{2} \cdots$} $\mathrm{O}_{2}, \mathrm{~N}_{2} \cdots \mathrm{N}_{2}$ e $\mathrm{O}_{2} \cdots \mathrm{N}_{2}$

No caso das espécies predominantes na atmosfera, $\mathrm{O}_{2}$ e $\mathrm{N}_{2}$, existem estudos experimentais e teóricos do desenvolvimento de superfícies de energia potencial, $\mathrm{SEP}$, para os dímeros de $\mathrm{O}_{2} \cdots \mathrm{O}_{2}{ }^{1,2}, \mathrm{~N}_{2} \cdots \mathrm{N}_{2}{ }^{3}$ e $\mathrm{O}_{2} \cdots$ $\mathrm{N}_{2}{ }^{4}$, no estado fundamental e excitado ${ }^{5}$. Estes estudos combinados, dados experimentais e modelo teórico, indicam que a maior parte das ligações destes dímeros é do tipo de van der Waals, repulsão + atração, e forças eletrostáticas, quadrupolo permanente - quadrupolo permanente. Por outro lado, as contribuições químicas, spin - spin, não são desprezíveis para sistemas do tipo $\mathrm{O}_{2}$ .. $\mathrm{O}_{2}$, camada aberta - camada fechada.

No caso destes dímeros de moléculas diatômicas homonucleares foi mostrado por V. Aquilanti e colaboradores $^{1,2}$ que a SEP pode ser definida em termos dos harmônicos esféricos após a separação da parte radial da parte angular. No caso do dímero do $\mathrm{O}_{2} \cdots \mathrm{N}_{2}{ }^{4}$ esta mesma expansão foi utilizada com sucesso. Esta forma de construção da SEP é muito interessante, pois mostra que para três dímeros diferentes, quanto à estrutura e ao tipo de ligação, foi possível utilizar a mesma expansão, reproduzindo os dados experimentais de geometria de equilíbrio e energia de interação. Este tipo de expansão para estas SEP's são fáceis de serem obtidas e a interpretação física dos seus termos a torna realística pois elas reproduzem informações micro e macroscópicas.

\section{SUPERFÍCIE DE ENERGIA POTENCIAL: $\mathrm{H}_{2} \mathrm{O} \cdots$ $\operatorname{Rg}$ e $\mathrm{H}_{2} \mathrm{~S} \cdots \mathrm{Rg}$}

Dos efeitos observáveis na química da atmosfera, as interações das espécies secundárias é a mais importante, e não somente de água com $\mathrm{O}_{2}, \mathrm{~N}_{2}$ e a própria $\mathrm{H}_{2} \mathrm{O}$, mas também com outras moléculas presentes na atmosfera, porém poucas informações se têm sobre estes sistemas e os quais ainda não são bem entendidos.

No estudo da interação de clusters de $\mathrm{H}_{2} \mathrm{O}$ com moléculas polares, $\left(\mathrm{H}_{2} \mathrm{O}\right)_{2},\left(\mathrm{H}_{2} \mathrm{O}\right)_{3}$ e $\mathrm{H}_{2} \mathrm{O} \cdots \mathrm{CO}^{6}$ e apolares, $\mathrm{H}_{2} \mathrm{O} \cdots \mathrm{H}_{2}, \mathrm{H}_{2} \mathrm{O} \cdots \mathrm{N}_{2}, \mathrm{H}_{2} \mathrm{O} \cdots \mathrm{O}_{2}, \mathrm{H}_{2} \mathrm{O} \cdots$ $\mathrm{CO}_{2}, \mathrm{H}_{2} \mathrm{O} \cdots \mathrm{CS}_{2}$ e $\mathrm{H}_{2} \mathrm{O} \cdots \mathrm{CH}_{4},{ }^{7}$ cálculos ab initio de otimização de geometria e determinação energia foram obtidos, mostrando que estas interações são maiores para o trímero e dímero de $\mathrm{H}_{2} \mathrm{O}$, seguidos dos clusters de $\mathrm{H}_{2} \mathrm{O}$ $\cdots \mathrm{CO}_{2}, \mathrm{H}_{2} \mathrm{O} \cdots \mathrm{N}_{2}, \mathrm{H}_{2} \mathrm{O} \cdots \mathrm{CH}_{4}$ e $\mathrm{H}_{2} \mathrm{O} \cdots \mathrm{H}_{2}$.

$\mathrm{O}$ papel da molécula de água na natureza é caracterizado por uma interação característica peculiar, as ligações de hidrogênio, sendo um dos fenômenos mais estudados tanto experimentalmente quanto teoricamente. Um dos clusters de água mais simples de serem modelados envolve os gases nobres, onde as interações eletrostáticas não estão presentes. Recentemente foram caracterizados experimentalmente os dímeros de $\mathrm{H}_{2} \mathrm{O}$-.. $\mathrm{Rg}(\mathrm{Rg}=\mathrm{He}, \mathrm{Ne}, \mathrm{Ar}, \mathrm{Kr}$ e Xe ) e construídas as SEP's teóricas ${ }^{8}$. Neste estudo observou que para os dímeros, do hélio ao xenônio, a distância O-Rg e a energia de interação aumentam, porém o ângulo $\mathrm{Rg}-\mathrm{OH}$ diminui. Neste estudo também se concluiu que as interações $\mathrm{H}_{2} \mathrm{O}$ .. Rg são essencialmente do tipo de van der Waals para os gases de baixo peso molecular, hélio e neônio, porém para os gases de maior peso molecular, como o xenônio, as energias são maiores do que as puramente de van der Waals.

Desta mesma série de compostos, foi estudado o $\mathrm{H}_{2} \mathrm{~S}$ $\cdots \mathrm{Rg}{ }^{9}$ e $\mathrm{H}_{2} \mathrm{~S} \cdots \mathrm{Xe}{ }^{10}$. A parte teórica deste trabalho, constou da determinação da geometria de equilíbrio 
para os complexos $\mathrm{H}_{2} \mathrm{~S} \cdots \mathrm{Rg}$, com $\mathrm{Rg}=\mathrm{He}, \mathrm{Ne}$, Ar e $\mathrm{Kr}$, e também a determinação da superfície de energia potencial em função da distância S-Rg e de três diferentes ângulos. Foi considerado o átomo de enxofre localizado na origem do sistema de coordenadas, a molécula de $\mathrm{H}_{2} \mathrm{~S}$ com simetria $\mathrm{C}_{2 \mathrm{v}}$ e os átomos de hidrogênio no plano $\mathrm{XY}$. O primeiro ângulo descreve a rotação no plano $\mathrm{XY}$, partindo do eixo de simetria $\mathrm{C}_{2 \mathrm{v}}$. $\mathrm{O}$ segundo é o ângulo fora do plano da molécula, variando no plano XZ, também partindo do eixo de simetria $\mathrm{C}_{2 \mathrm{v}}$ e o terceiro, descreve a rotação no plano $\mathrm{YZ}$, partindo do eixo $\mathrm{Z}$. Com isto foi possível estudar a anisotropia do sistema. Por este estudo foi possível mostrar que indo do $\mathrm{He}$ para o $\mathrm{Kr}$ a distância de equilíbrio aumenta em $22 \%$ enquanto que a energia aumenta em $7 \%$, como observado para o $\mathrm{H}_{2} \mathrm{O} \cdots \mathrm{Rg}^{8}$.

\section{Peróxidos e Persulfetos}

Um estudo sistemático quanto às estruturas $\mathrm{e}$ propriedades elétricas do $\mathrm{H}_{2} \mathrm{O}_{2}{ }^{11} \mathrm{e}_{2} \mathrm{~S}_{2}{ }^{12}$, e também com a substituição do hidrogênio por grupos alquil ${ }^{14} \mathrm{e}$ átomos da família dos halogênios ${ }^{15}$ foi conduzido no intuito de identificar o melhor nível de cálculo que reproduz os dados experimentais. A ênfase foi dada para o ângulo de torsão, ao longo da ligação OO ou SS, conforme o caso, que conduz a quiralidade, mudança de estereoisomeria.

$\mathrm{O}$ entendimento da dinâmica envolvida nestas ligações fracas, OO e SS, é importante, pois ela tem um papel fundamental em moléculas e clusters de relevância não só de interesse atmosférico ${ }^{16,}{ }^{17,}{ }^{18}$, mas também em outras áreas tais como: combustão e processos bioquímicos. Estas ligações são geralmente mais longas e mais fracas do que as apresentadas em ambientes orgânicos onde elas ocorrem, e exibem uma dinâmica particular que se manifestam em fenômenos intramolecular e intermolecular, que serão discutidos na seção seguinte. Os fenômenos intramoleculares são cineticamente identificados como isomerizações, enquanto que espectroscopicamente são referenciados por uma ampla faixa de modos anarmônicos. Os fenômenos intermoleculares têm sido amplamente estudados, como ligações de hidrogênio semelhantes as que ocorrem com a água, em dímeros e clusters maiores.

\section{SUPERFÍCIE DE ENERGIA POTENCIAL PARA SISTEMAS DE CINCO CORPOS}

Na literatura existem vários trabalhos que descrevem a interação de $\mathrm{H}_{2} \mathrm{O}_{2}$ com várias moléculas e íons, tais como $\mathrm{F}^{-}, \mathrm{Cl}^{-}, \mathrm{Br}^{-}, \mathrm{Li}^{+}, \mathrm{Na}^{+}[19], \mathrm{NO}^{+}, \mathrm{CN}^{-}, \mathrm{HCN}, \mathrm{HNC}$, $\mathrm{CO}^{20}, \mathrm{~N}_{2}, \mathrm{He}, \mathrm{Ne}, \mathrm{Ar}^{21}$ e clusters de $\left(\mathrm{H}_{2} \mathrm{O}_{2}\right) \mathrm{n}$ com $\mathrm{n}=1-$ 15,22 e $28{ }^{22}$, porém nenhum destes propõem um modelo teórico geral para a superfície de energia potencial, SEP. Nós propomos um modelo para a interação do $\mathrm{H}_{2} \mathrm{X}_{2} \quad \cdots$ $\mathrm{Rg}$, com $\mathrm{Rg}=\mathrm{He}, \mathrm{Ne}, \mathrm{Ar}, \mathrm{Kr}$ e $\mathrm{Xe}$ e $\mathrm{X}=\mathrm{O}^{13}$ e S ${ }^{12}$, que envolve cinco átomos, em que os modos intramolecular do peróxido e persulfeto de hidrogênio são considerados congelados exceto pelo ângulo torsional $\gamma$ (ver Figura 1 (a) e (b)). É mostrado que o potencial de interação neste caso pode ser expresso em função de quatro variáveis: a coordenada polar $\vec{r}$, os ângulos $\alpha$ e $\beta$ (mostrado na Figura 1(b)) e o ângulo de diedro $\gamma$. Desde que a faixa das variáveis angulares tenha três dimensões isomórficas para $\mathrm{S}^{3}$ (coordenadas esféricas embebidas num espaço euclidiano de quatro dimensões $-\mathfrak{R}^{4}$ ), é apropriado usar uma expansão ortonormal em termos dos harmônicos hiperesféricos. Este conjunto de bases é definido em termos de combinações da função D-Wigner, que pode ser encontrada tabelada na literatura ${ }^{23}$.

\section{Coordenadas e Simetria}

A posição do átomo de gás nobre em relação à molécula de $\mathrm{H}_{3} \mathrm{O}_{2}$ é expressa em termos da coordenada esférica polar $\stackrel{\mathrm{r}}{=}(\mathrm{r}, \alpha, \beta)$, onde $\alpha$ e $\beta$ são os ângulos azimutal e polar respectivamente [figura 1 (b)]. A origem é o centro de massa instantâneo da molécula, que coincide com o meio da ligação $\mathrm{OO}$ para a configuração trans. Considerando o eixo z como sendo paralelo ao da ligação OO e o ângulo torsional $\gamma$. Pode-se definir as coordenadas $(\mathrm{x}, \mathrm{y}, \mathrm{z})$ em função dos ângulos $\alpha \mathrm{e} \beta$ e a distância $r$ a partir do centro de massa como sendo: $x=r \sin \beta \sin \alpha$; $y=r \sin$ $\beta \cos \alpha ; z=r \cos \beta$, com $0 \leq \mathrm{r} \leq \infty, 0 \leq \beta \leq \pi, 0 \leq \alpha \leq 2 \pi$, e o vetor $\overrightarrow{\mathrm{r}}=(\mathrm{r}, \alpha, \beta)$ um vetor de Jacobi, de tal forma que sua representação será útil para o tratamento dinânico.

O potencial descrito para o sistema depende destas quatro coordenadas, podendo facilmente ser observadas algumas propriedades de simetria.

$$
V(r ; \alpha, \beta, \gamma)=V(r ;-\alpha, \beta,-\gamma)=V(r ;-\alpha, \pi-\beta, \gamma)=V(r ; \alpha, \pi-\beta,-\gamma)
$$

A forma analítica da SEP que será construída poderá ser dividida em duas contribuições: 


$$
V(r ; \alpha, \beta, \gamma)=V_{e x t}(r ; \alpha, \beta)+V_{\text {int }}(r ; \gamma)
$$

onde o termo $\mathrm{V}_{\text {ext }}(\mathrm{r} ; \alpha, \beta)$ inclui as contribuições dependentes da distância relativa ao átomo - molécula e sua orientação, e o $\mathrm{V}_{\text {int }}(\mathrm{r} ; \gamma)$ é o termo intramolecular que depende da distância r e do ângulo de torsão $\gamma$.

\section{Expansão em Harmônicos Hiperesféricos}

A SEP, equação (2), pode ser expressa em termos de uma série apropriada de funções angulares multiplicadas pelo coeficiente radial (momentos). Um conjunto completo adequado de funções D-Wigner, que depende de três ângulos de Euler com o mesmo domínio dos ângulos esféricos $\alpha, \beta$ e do ângulo de torsão $\gamma$.

As funções D-Wigner são em geral complexas ${ }^{23}$, portanto uma forma alternativa, denominada $\mathfrak{R}_{\mathrm{MM}}{ }^{\mu}$ $(\alpha, \beta, \gamma)$ impondo que a função seja real será utilizada, de tal forma que:

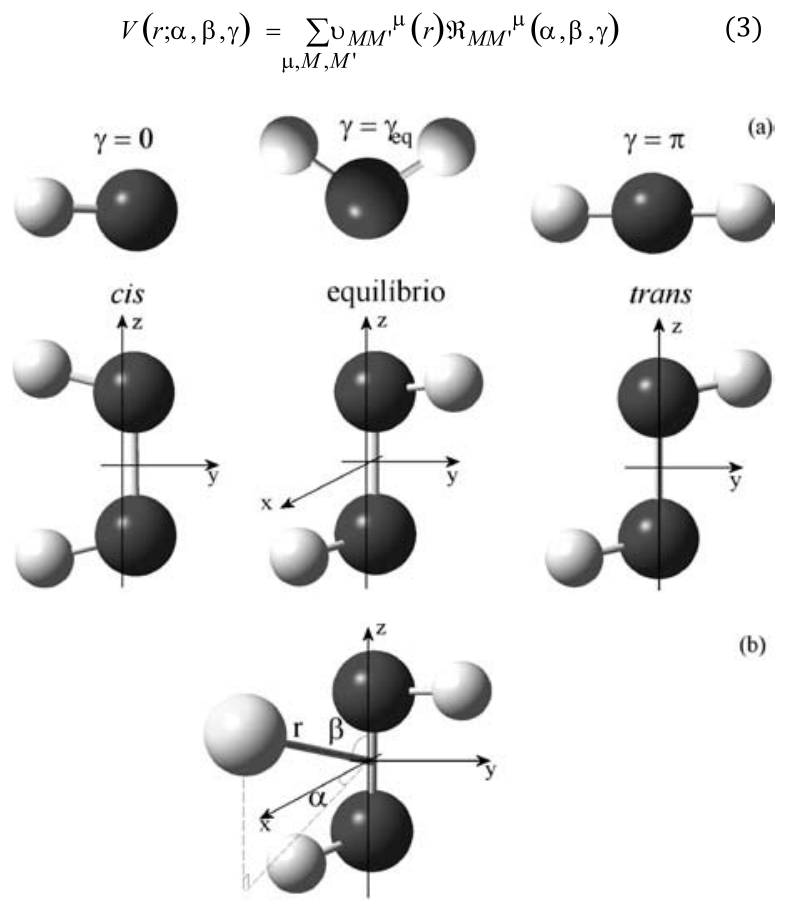

Figura 1: (a) Ilustração das três configurações características para o peróxido de hidrogênio: cis, equilibrio e trans. O ângulo torsional, denominado por $\gamma$, ao redor da ligação $\mathrm{OO}$, ângulo de diedro $\mathrm{HOOH}$, também são indicados em cada caso. (b) Definição da coordenada esférica $\overrightarrow{\mathrm{r}}=(\mathrm{r}, \alpha, \beta)$, especificando a posição do gás nobre de acordo com as configurações da parte (a) da figura. A distância $\vec{r}$ vai do $\operatorname{Rg}$ até o centro de massa da molécula do $\mathrm{H}_{2} \mathrm{O}_{2}, \beta$ é o ângulo entre $\vec{r}$ e o plano $z$ e $\alpha$ é o ângulo entre o eixo $x$ e a projeção de $\vec{r}$ no plano $x y$ onde $\mu=0,1,2, \cdots$ e $\mathrm{M}\left(\mathrm{M}^{\prime}\right)=-\mu,-\mu+1, \cdots,-1,0,1, \cdots, \mu-1, \mu$ e os coeficientes de $v_{M M}{ }^{\mu}(r)$ são expansões de momentos que dependem somente da coordenada $r$. Os termos reais da função $D$-Wigner, $\mathfrak{R}_{\mathrm{MM}}{ }^{\mu}(\alpha, \beta, \gamma)$ são escritos como combinações lineares da função complexa D-Wigner:

$$
\Re_{M M^{\mu}}{ }^{\mu}(\alpha, \beta, \gamma)=\sqrt{\frac{8 \pi^{2}}{2(2 \mu+1)}}\left(D_{M M^{,}}{ }^{\mu}(\alpha, \beta, \gamma)+\varepsilon D_{-M-M}{ }^{\mu}(\alpha, \beta, \gamma)\right)
$$

$$
\Re_{-M-M^{,}}{ }^{\mu}(\alpha, \beta, \gamma)=\sqrt{\frac{8 \pi^{2}}{2(2 \mu+1)}}\left(\varepsilon D_{M M^{\mu}}{ }^{\mu}(\alpha, \beta, \gamma)-D_{-M-M},{ }^{\mu}(\alpha, \beta, \gamma)\right)
$$

onde $\varepsilon=(-1)^{M-M^{\prime}}$, e $D_{M M^{\prime}}{ }^{\mu}(\alpha, \beta, \gamma)=e^{i M \alpha} d_{M M^{\prime}}{ }^{\mu}(\beta) e^{i M^{\prime} \gamma}$.

Em particular:

$$
\Re_{00}{ }^{0}=D_{00}{ }^{0}=d_{00}{ }^{0}
$$

onde $\Re_{00}^{0}$ é essencialmente um polinômio de Legendre em $\cos (\beta)$.

Para $\mu=0, \Re_{00}{ }^{0}(\alpha, \beta, \gamma)=1$. Para $\mu=1$ e $\mu=2$ existem 34 funções que são listadas na tabela 1. A maioria destas funções pode ser eliminada devido à condição de simetria, equação (1), na qual as funções que dependem de $\sin ^{\mathrm{n}}(\alpha), \sin ^{\mathrm{n}}(\gamma)$ e $\cos ^{\mathrm{n}}(\beta)$, onde $n$ é um número inteiro impar, podem ser descartadas. Após impor a condição de simetria, o conjunto de base se reduz a seis funções, $\mathfrak{R}_{00}{ }^{0}, \mathfrak{R}_{10}{ }^{1}, \mathfrak{R}_{01}{ }^{1}, \mathfrak{R}_{00}{ }^{2}, \mathfrak{R}_{20}{ }^{2}$ e $\mathfrak{R}_{02}{ }^{2}$, que correspondem aos coeficientes da equação (3).

Em geral, o uso de potenciais selecionados apropriadamente para configurações de ângulos prédeterminados $\gamma$, em função da distancia $r$ do átomo para o centro de massa da molécula, correspondentes a aproximações em diferentes direções (valores específicos para $\alpha$ e $\beta$ ), nos permite montar um sistema de equações lineares, cuja solução fornece a SEP completa do sistema.

\section{CONFIGURAÇÕES PRINCIPAIS E EXPANSÃO DE MOMENTOS}

A escolha das configurações recai nas considerações físicas das características geométricas do sistema. Foram consideradas as três configurações geométricas para o $\mathrm{H}_{2} \mathrm{X}_{2}$ correspondentes a de equilíbrio, $\gamma=\gamma_{\text {eq }}$, e a cis e a trans, $\operatorname{com} \gamma=0$ e $\gamma=\pi$, respectivamente. 
Tabela 1: Harmônicos hiperesféricos reais, $\mathfrak{R}_{\mathrm{MM}}{ }^{\mu}{ }^{\mu}(\alpha, \beta, \gamma)$, para $\mu=1$ e $\mu=2$

\begin{tabular}{|c|c|c|c|c|c|}
\hline \multicolumn{6}{|c|}{$\mu=1$} \\
\hline $\mathrm{M} / \mathrm{M}^{\prime}$ & \multicolumn{2}{|l|}{1} & 0 & \multicolumn{2}{|r|}{-1} \\
\hline 1 & \multicolumn{2}{|c|}{$\frac{1+\cos (\beta)}{\sqrt{2}}(\cos \alpha \cos \gamma-\sin \alpha \sin \gamma)$} & $-\sin \beta \cos \alpha$ & \multicolumn{2}{|c|}{$\frac{1-\cos (\beta)}{\sqrt{2}}(\cos \alpha \sin \gamma-\sin \alpha \cos \gamma)$} \\
\hline 0 & \multicolumn{2}{|l|}{$\sin \beta \cos \gamma$} & $\cos \beta$ & \multicolumn{2}{|r|}{$-\sin \beta \sin \gamma$} \\
\hline-1 & \multicolumn{2}{|c|}{$\frac{1-\cos (\beta)}{\sqrt{2}}(\cos \alpha \cos \gamma+\sin \alpha \sin \gamma)$} & $\sin \alpha \sin \beta$ & \multicolumn{2}{|c|}{$\frac{1+\cos (\beta)}{\sqrt{2}}(\sin \alpha \cos \gamma+\cos \alpha \sin \gamma)$} \\
\hline \multicolumn{6}{|c|}{$\mu=2$} \\
\hline $\mathrm{M} / \mathrm{M}^{\prime}$ & 2 & 1 & 0 & -1 & -2 \\
\hline 2 & $\begin{array}{l}\frac{(1+\cos \beta)^{2}}{2 \sqrt{2}} \times \\
\times[\cos (2 \alpha) \cos (2 \gamma)-\sin (2 \alpha) \sin (2 \gamma)]\end{array}$ & $\begin{array}{l}\frac{\sin \beta(1+\cos \beta)}{\sqrt{2}} \times \\
\times[\sin (2 \alpha) \sin \gamma-\cos (2 \alpha) \cos \gamma]\end{array}$ & $\frac{\sqrt{3}}{2} \sin ^{2} \beta \cos (2 \alpha)$ & $\begin{array}{l}-\frac{\sin \beta(1-\cos \beta)}{\sqrt{2}} \times \\
\times[\cos (2 \alpha) \cos \gamma-\sin (2 \alpha) \sin \gamma]\end{array}$ & $\begin{array}{l}\frac{(1-\cos \beta)^{2}}{22} \times \\
\times[\cos (2 \alpha) \sin (2 \gamma)-\sin (2 \alpha) \cos (2 \gamma)]\end{array}$ \\
\hline 1 & $\begin{array}{l}\frac{\sin \beta(1+\cos \beta)}{\sqrt{2}} \times \\
\times[\cos \alpha \cos (2 \gamma)-\sin \alpha \sin (2 \gamma)]\end{array}$ & $\begin{array}{l}\frac{2 \cos ^{2} \beta+\cos \beta-1}{\sqrt{2}} \times \\
\times(\cos \alpha \cos \gamma-\sin \alpha \sin \gamma)\end{array}$ & $-\sqrt{3} \sin \beta \cos \beta \cos \alpha$ & $\begin{array}{l}\frac{2 \cos ^{2} \beta+\cos \beta-1}{\sqrt{2}} \times \\
\times(\cos \alpha \sin \gamma-\sin \alpha \cos \gamma)\end{array}$ & $\begin{array}{l}\frac{\sin \beta(1-\cos \beta)}{\sqrt{2}} \times \\
\times[\sin \alpha \cos (2 \gamma)-\cos \alpha \sin (2 \gamma)]\end{array}$ \\
\hline 0 & $\frac{\sqrt{3}}{2} \sin ^{2} \beta \cos (2 \gamma)$ & $\sqrt{3} \sin \beta \cos \beta \cos \gamma$ & $\frac{3 \cos ^{2} \beta-1}{2}$ & $-\sqrt{3} \sin \beta \cos \beta \sin \gamma$ & $\frac{\sqrt{3}}{2} \sin ^{2} \beta \sin (2 \gamma)$ \\
\hline-1 & $\begin{array}{l}\frac{\sin \beta(1-\cos \beta)}{\sqrt{2}} \times \\
\times[\cos \alpha \cos (2 \gamma)-\sin \alpha \sin (2 \gamma)]\end{array}$ & $\begin{array}{l}\frac{2 \cos ^{2} \beta+\cos \beta-1}{\sqrt{2}} \times \\
\times(\cos \alpha \cos \gamma-\sin \alpha \sin \gamma)\end{array}$ & $\sqrt{3} \sin \beta \cos \beta \sin \alpha$ & $\begin{array}{l}\frac{2 \cos ^{2} \beta+\cos \beta-1}{\sqrt{2}} \times \\
\times(\cos \alpha \sin \gamma+\sin \alpha \cos \gamma)\end{array}$ & $\begin{array}{l}\frac{-\sin \beta(1+\cos \beta)}{\sqrt{2}} \times \\
\times[\sin \alpha \cos (2 \gamma)+\cos \alpha \sin (2 \gamma)]\end{array}$ \\
\hline-2 & $\begin{array}{l}\frac{(1-\cos \beta)^{2}}{2 \sqrt{2}} \\
{[\cos (2 \alpha) \cos (2 \gamma)+\sin (2 \alpha) \sin (2 \gamma)]}\end{array}$ & $\begin{array}{l}\frac{\sin \beta(1-\cos \beta)}{\sqrt{2}} \times \\
\times[\sin (2 \alpha) \cos \gamma-\cos (2 \alpha) \sin \gamma]\end{array}$ & $\frac{\sqrt{3}}{2} \sin ^{2} \beta \sin (2 \alpha)$ & $\begin{array}{l}\frac{\sin \beta(1+\cos \beta)}{\sqrt{2}} \times \\
\times[\sin (2 \alpha) \cos \gamma-\cos (2 \alpha) \sin \gamma]\end{array}$ & $\begin{array}{l}\frac{(1+\cos \beta)^{2}}{2 \sqrt{2}} \times \\
\times[\sin (2 \alpha) \cos (2 \gamma)+\cos (2 \alpha) \sin (2 \gamma)]\end{array}$ \\
\hline
\end{tabular}

Para cada uma destas configurações foi considerado o átomo movendo ao longo da direção da ligação $\mathrm{XX}(\beta=0$ e $\alpha$ indeterminado) e movendo na direção perpendicular a esta ligação $(\beta=\pi / 2) \operatorname{com} \alpha=0, \pi / 2$ e p. Este conjunto de onze configurações compõe as configurações principais e são listadas na Tabela 2. Os momentos são então obtidos via combinação linear dos potenciais calculados para cada configuração.

A representação da energia potencial como sendo a soma de contribuições intermolecular e intramolecular [equação (2)] pode ser obtida em termos dos harmônicos hiperesféricos, $\Re_{0 \mathrm{M}}{ }^{\mu}(0, \pi / 2, \gamma)$, expandindo os dois termos da equação (2) individualmente. $O$ termo
Tabela 2: Definição das configurações principais, em termos dos ângulos $\alpha, \beta$ e $\gamma$

\begin{tabular}{|c|c|c|c|c|c|}
\hline & & \multicolumn{3}{|c|}{$\beta=\pi / 2$} & $\beta=0$ \\
\hline & $\gamma$ & $\alpha=0$ & $\alpha=\pi / 2$ & $\alpha=\pi$ & $\alpha=$ ind. \\
\hline cis & $0^{\circ}$ & $C>$ & $C \perp$ & $C^{<}$ & $C \|$ \\
\hline equilíbrio & $\gamma_{e q}$ & $E^{>}$ & $E \perp$ & $E^{<}$ & $E \|$ \\
\hline trans & $\pi$ & $T>$ & $T \perp$ & $T<$ & $E \|$ \\
\hline
\end{tabular}


intramolecular, $\mathrm{V}_{\text {int }}(r ; \gamma)$, envolve as funções $\Re_{0 M}{ }^{\mu}(0,0, \gamma)$ pois são independentes de $\alpha$ e $\beta$. Enquanto que o termo intermolecular, $\mathrm{V}_{\text {ext }}(r ; \alpha, \beta)$, envolve somente os termos $\mathfrak{R}_{M 0}{ }^{\mu}{ }^{\mu}(\alpha, \beta, \gamma)$. Entretanto, apesar de não estar explicito a dependência de $\gamma$ no $\mathrm{V}_{\text {ext }}(r ; \alpha, \beta)$, ele é obtido para as três configurações do $\mathrm{H}_{2} \mathrm{O}_{2}$ (cis, equilíbrio e trans) e logo depende parametricamente de $\gamma$.

Os momentos $\mathrm{U}_{\text {мм }}{ }^{\mu}(i ; r)$, onde a dependência da geometria molecular esta especificada no símbolo $i=\mathrm{C}$, $E$ ou $T$, são calculados pelo potencial correspondente as configurações principais, tais como:

$$
\begin{gathered}
V_{<}(i ; r)=\mathrm{v}_{00}^{0}(i ; r)+\mathrm{v}_{10}^{1}(i ; r) \Re_{10}^{1}\left(\pi, \pi / 2, \gamma_{i}\right)+ \\
+\mathrm{v}_{00}^{2}(i ; r) \Re_{00}^{2}\left(\pi, \pi / 2, \gamma_{i}\right)+\mathrm{v}_{20}^{2}(i ; r) \Re_{20}^{2}\left(\pi, \pi / 2, \gamma_{i}\right) \\
V_{\perp}(i ; r)=\mathrm{v}_{00}^{0}(i ; r)+\mathrm{v}_{10}^{1}(i ; r) \Re_{10}^{1}\left(\pi / 2, \pi / 2, \gamma_{i}\right)+ \\
+\mathrm{v}_{00}^{2}(i ; r) \Re_{00}^{2}\left(\pi / 2, \pi / 2, \gamma_{i}\right)+\mathrm{v}_{20}^{2}(i ; r) \Re_{20}^{2}\left(\pi / 2, \pi / 2, \gamma_{i}\right) \\
V_{>}(i ; r)=\mathrm{v}_{00}^{0}(i ; r)+\mathrm{v}_{10}^{1}(i ; r) \Re_{10}^{1}\left(0, \pi / 2, \gamma_{i}\right)+ \\
+\mathrm{v}_{00}^{2}(i ; r) \Re_{00}^{2}\left(0, \pi / 2, \gamma_{i}\right)+\mathrm{v}_{20}^{2}(i ; r) \Re_{20}^{2}\left(0, \pi / 2, \gamma_{i}\right) \\
V_{\|}(i ; r)=\mathrm{v}_{00}^{0}(i ; r)+\mathrm{v}_{10}^{1}(i ; r) \Re_{10}^{1}\left(i n d, 0, \gamma_{i}\right)+ \\
+\mathrm{v}_{00}^{2}(i ; r) \Re_{00}^{2}\left(i n d, 0, \gamma_{i}\right)+\mathrm{v}_{20}^{2}(i ; r) \Re_{20}^{2}\left(i n d, 0, \gamma_{i}\right)
\end{gathered}
$$

que pode ser invertido para:

$$
\begin{aligned}
& \nu_{00}^{0}(i ; r)=\frac{1}{6} V_{<}(i ; r)+\frac{1}{3} V_{\perp}(i ; r)+\frac{1}{6} V_{>}(i ; r)+\frac{1}{3} V_{\|}(i ; r) \\
& U_{10}^{1}(i ; r)=\frac{1}{2} V_{<}(i ; r)-\frac{1}{2} V_{>}(i ; r) \\
& \nu_{00}^{2}(i ; r)=-\frac{1}{6} V_{<}(i ; r)-\frac{1}{3} V_{\perp}(i ; r)-\frac{1}{6} V_{>}(i ; r)+\frac{2}{3} V_{\|}(i ; r) \\
& \nu_{20}^{2}(i ; r)=\frac{\sqrt{3}}{6} V_{<}(i ; r)+\frac{\sqrt{3}}{3} V_{\perp}(i ; r)+\frac{\sqrt{3}}{6} V_{>}(i ; r)
\end{aligned}
$$

o termo $\mathrm{v}_{10}{ }^{1}(T ; r)=0$, pois o $\mathrm{V}<(T ; r)=\mathrm{V}>(T ; r)$.

É conveniente definir um coeficiente wi $(\gamma)$, que estabelece um peso para cada configuração principal $(i$ $=C, E$ e $T$ ) de acordo com o valor correspondente de $\gamma$. Supondo o potencial torsional expandido numa série de cossenos, incluindo os termos $\cos (n \gamma), \operatorname{com} n=0,1$ e 2 , tem-se:

$$
w_{i}(\gamma)=a_{i}+b_{i} \cos (\gamma)+c_{i} \cos (2 \gamma)
$$

de tal forma que:

$$
\begin{array}{lll}
w_{C}(0)=1 & w_{C}(\pi)=0 & w_{C}\left(\gamma_{e q}\right)=0 \\
w_{T}(0)=0 & w_{T}(\pi)=1 & w_{T}\left(\gamma_{e q}\right)=0 \\
w_{E}(0)=0 & w_{E}(\pi)=0 & w_{E}\left(\gamma_{e q}\right)=1
\end{array}
$$

de onde se obtêm:

$$
\begin{aligned}
& w_{C}(\gamma)=-\frac{\cos \gamma_{e q}+\cos 2 \gamma_{e q}}{4 \sin ^{2} \gamma_{e q}}+\frac{1}{2} \cos \gamma+\frac{\left(1+\cos \gamma_{e q}\right)}{4 \sin ^{2} \gamma_{e q}} \cos 2 \gamma \\
& w_{T}(\gamma)=\frac{\cos \gamma_{e q}-\cos 2 \gamma_{e q}}{4 \sin ^{2} \gamma_{e q}}-\frac{1}{2} \cos \gamma+\frac{\left(1+\cos \gamma_{e q}\right)}{4 \sin ^{2} \gamma_{e q}} \cos 2 \gamma \\
& w_{E}(\gamma)=\frac{1}{2 \sin ^{2} \gamma_{e q}}-\frac{1}{2 \sin ^{2} \gamma_{e q}} \cos 2 \gamma
\end{aligned}
$$

Agora se pode escrever a expressão geral para o $\mathrm{V}_{\text {ext }}(r ; \alpha, \beta, \gamma)$ como sendo:

$V_{\text {ext }}(r ; \alpha, \beta, \gamma)=\sum_{i} w_{i}(\gamma)\left[v_{10}^{1}(i ; r) \Re_{10}^{1}+v_{00}^{2}(i ; r) \Re_{00}^{2}+v_{20}^{2}(i ; r) \Re_{20}^{2}\right]$

onde os momentos determinados nas Equações 8, são utilizados para obter o potencial intramolecular, exceto o termo $v_{00}{ }^{0}(\mathrm{i} ; \mathrm{r})$, que é utilizado para obter o potencial intermolecular, $\mathrm{V}_{\mathrm{int}}(r ; \alpha, \beta, \gamma)$, que será dado por:

$$
V_{\text {int }}(r ; \gamma)=\mathrm{v}_{00}^{0}(r)+\mathrm{v}_{01}^{1}(r) \Re_{01}^{1}(0, \pi / 2, \gamma)+\mathrm{v}_{02}^{2}(r) \Re_{02}^{2}(0, \pi / 2, \gamma)
$$

de onde se pode concluir que:

$$
\begin{aligned}
& \overline{\mathrm{v}}_{00}^{0}(r)=-\frac{2 \mathrm{v}_{00}^{0}(E ; r)+\left(\mathrm{v}_{00}^{0}(C ; r)-\mathrm{v}_{00}^{0}(T ; r)\right) \cos \gamma+\left(\mathrm{v}_{00}^{0}(C ; r)-\mathrm{v}_{00}^{0}(T ; r)\right) \cos 2 \gamma}{4 \cos ^{2} \gamma} \\
& \overline{\mathrm{v}}_{01}^{1}(r)=0.5 \mathrm{v}_{00}^{0}(C ; r)-0.5 \mathrm{v}_{00}^{0}(T ; r) \\
& \overline{\mathrm{v}}_{02}^{2}(r)=\frac{\mathrm{v}_{00}^{0}(C ; r)-2 \mathrm{v}_{00}^{0}(E ; r)\left(\mathrm{v}_{00}^{0}(C ; r)-\mathrm{v}_{00}^{0}(T ; r)\right) \cos \gamma}{2 \sqrt{3} \cos ^{2} \gamma}
\end{aligned}
$$

$\mathrm{O}$ momento $\bar{v}_{00}{ }^{0}(r)$ representa o potencial médio sobre todos os ângulos $\alpha$ e $\beta$, enquanto que os termos $\bar{v}_{01}{ }^{1}(r)$ e $\bar{v}_{02}^{2}(r)$ representam a anisotropia do sistema.

\section{Resultados}

Estas energias ab initio foram determinadas usando o programa Gaussian03 ${ }^{24}$. A geometria dos monômeros $\mathrm{H}_{2} \mathrm{X}_{2}$ com $\mathrm{X}=\mathrm{O}$ e $\mathrm{S}$ foi mantida congelada na sua geometria de equilíbrio, exceto pelo ângulo $\gamma$, conforme Tabela 3. De acordo com estudos anteriores 11,12 verificou-se que cálculos de Møller-Plesset de segunda ordem em aug-cc-pVTZ reproduzem bem os dados experimentais de geometrias e barreiras sem comprometer consideravelmente o tempo computacional, portanto este nível foi escolhido para o $\mathrm{He}, \mathrm{Ne}, \mathrm{Ar}$ e $\mathrm{Kr}$ e para o Xe 
Tabela 3: Geometrias e Barreiras para o $\mathrm{H}_{2} \mathrm{X}_{2} \operatorname{com} \mathrm{X}=\mathrm{O}$ e S, em MP2/aug-cc-pVTZ

\begin{tabular}{|c|c|c|c|c|c|c|}
\hline & configuração & $\mathrm{R}_{\mathrm{HX}}[\AA]$ & $\mathrm{R}_{\mathrm{XX}}[\AA]$ & $\angle_{\mathrm{HXX}}\left[^{\circ}\right]$ & $\angle_{\mathrm{HXXH}}\left[^{\circ}\right]$ & $\begin{array}{c}\text { Barreira } \\
{\left[\mathrm{kcal} \mathrm{mol}^{-1}\right]}\end{array}$ \\
\hline \multirow{9}{*}{$\mathrm{H}_{2} \mathrm{O}_{2}$} & \multirow{3}{*}{ equilibrio } & 0.9668 & 1.4537 & 99.6128 & 112.5422 & \multirow{3}{*}{0.0} \\
\hline & & $(0.950 \pm 0.005)^{\mathrm{a}}$ & $(1.475 \pm 0.004)^{\mathrm{a}}$ & $(94.8 \pm 2)^{\mathrm{a}}$ & $(119.8 \pm 3)^{\mathrm{a}}$ & \\
\hline & & $(0.9627)^{b}$ & $(1.4525)^{b}$ & $(99.91)^{b}$ & $(112.46)^{b}$ & \\
\hline & \multirow{3}{*}{ cis } & 0.9670 & 1.4628 & 104.1053 & 0.0000 & 7.3623 \\
\hline & & $(0.9628)^{\mathrm{b}}$ & $(1.4630)^{\mathrm{b}}$ & $(104.14)^{\mathrm{b}}$ & $(0.0)^{\mathrm{b}}$ & $(7.276)^{\mathrm{b}}$ \\
\hline & & & & & & $(7.033 \pm 0.071)^{\mathrm{c}}$ \\
\hline & \multirow{3}{*}{ trans } & 0.9663 & 1.4652 & 97.9109 & 180.0 & 1.1036 \\
\hline & & $(0.9616)^{\mathrm{b}}$ & $(1.4637)^{\mathrm{b}}$ & $(98.31)^{b}$ & $(180.0)^{\mathrm{b}}$ & $(1.078)^{b}$ \\
\hline & & & & & & $(1.104 \pm 0.011)^{\mathrm{c}}$ \\
\hline \multirow{8}{*}{$\mathrm{H}_{2} \mathrm{~S}_{2}$} & \multirow{2}{*}{ equilibrio } & 1.3357 & 2.0574 & 97.7112 & & \multirow{2}{*}{0.0} \\
\hline & & $1.3421^{\mathrm{d}}$ & $2.0610^{\mathrm{d}}$ & $97.51^{d}$ & 91.05 & \\
\hline & \multirow{3}{*}{ Cis } & 1.3317 & 2.1105 & 96.0634 & $90.76^{\mathrm{d}}$ & 8.2154 \\
\hline & & & & & \multirow{2}{*}{0.0} & $7.84^{\mathrm{d}}$ \\
\hline & & & & & & $7.5 \pm 0.15^{\mathrm{e}}$ \\
\hline & \multirow{3}{*}{ Trans } & 1.3333 & 2.0990 & 92.4978 & & 5.7272 \\
\hline & & & & & \multirow[b]{2}{*}{180.0} & $5.02^{\mathrm{d}}$ \\
\hline & & & & & & $5.0 \pm 0.15^{\mathrm{e}}$ \\
\hline
\end{tabular}

Nota: a dado experimental [28] ${ }^{\mathrm{b}}$ dado teórico [29] em $\left.\operatorname{CCSD}(\mathrm{T}) / \mathrm{cc}-\mathrm{pVQZ}\right\}{ }^{\mathrm{c}}$ dado experimental [30] d dado experimental [31] ${ }^{\mathrm{e}}$ dado teórico [32]

utilizou-se a base aug-cc-pVTZ-PP ${ }^{25,26}$. Com o intuito de minimizar o erro de superposição de bases, o método de Boys e Bernardi foi empregado ${ }^{27}$. A energia de interação foi calculada usando a técnica supermolecular:

$$
E_{C P}=E_{A B}\left(\chi_{A}+\chi_{B}\right)-\left[E_{A}\left(\chi_{A}+\chi_{B}\right)+E_{B}\left(\chi_{A}+\chi_{B}\right)\right]
$$

onde $\chi_{A}$ e $\chi_{B}$ são as bases de cada monômero do complexo AB. Utilizando os pontos ab initio, foi determinada uma função analítica para cada uma das configurações principais, segundo a função de Rydberg generalizada de quinto grau:

$$
V(r)=D_{e} \sum_{j=1}^{5}\left[1+a_{j}\left(r-r_{e q}\right)^{j}\right] \exp \left[-a_{1}\left(r-r_{e q}\right)\right]+E_{r e f}
$$

onde $D_{\mathrm{e}}, r_{\text {eq }}, E_{\text {ref }}$ e ai são os parâmetros ajustados, via técnicas não lineares tal que o erro entre as energias $a b$ initio e as analíticas sejam mínimos. Utilizando a função analítica para cada caso nas equações de (2), (12) e (13) é possível determinar o potencial total do sistema.

\section{Sistema $\mathrm{H}_{2} \mathrm{O}_{2} \cdots \mathbf{R g}$}

Foram calculadas 81 energias ab initio para as onze configurações principais, conforme Tabela 2, para o $\mathrm{H}_{2} \mathrm{O}_{2}$ $\cdots$ He e $\mathrm{H}_{2} \mathrm{O}_{2} \cdots \mathrm{Ne}, 92$ pontos para o $\mathrm{H}_{2} \mathrm{O}_{2} \cdots$ Ar e $\mathrm{H}_{2} \mathrm{O}_{2}$ $\cdots \mathrm{Kr}$ e 131 para $\mathrm{o}_{2} \mathrm{O}_{2} \cdots$ Xe. Todas as energias foram obtidas em função da distância $r$ [figura 1b] entre o gás nobre e o centro de massa da molécula de $\mathrm{H}_{2} \mathrm{O}_{2}$.

As Figuras de $2-6$ comparam as energias ab initio 
com as obtidas via o modelo proposto para o $\mathrm{He}, \mathrm{Ne}$, $\mathrm{Ar}, \mathrm{Kr}$ e $\mathrm{Xe}$, respectivamente. $\mathrm{O}$ primeiro fato que chama a atenção nessas figuras é que as energias estão agrupadas em três regiões: a de equilíbrio, inferior, a trans, intermediária, e a cis a superior, e que é comum para todos os sistemas analisados.

Outra tendência comum que pode ser observada é que a distância do mínimo e a energia de interação aumentam quando se parte de um átomo leve, He, para um átomo pesado, Xe. Este comportamento também foi observado para outros sistemas, tais como o $\mathrm{H}_{2} \mathrm{O} \cdots \mathrm{Rg}^{8}$ e $\mathrm{H}_{2} \mathrm{~S} \cdots$ $\operatorname{Rg}{ }^{9,10}$.

Utilizando uma configuração teste, para a geometria de equilíbrio $\left(\gamma=112.54^{\circ}\right)$ com $\alpha=\gamma_{\mathrm{eq}} / 2$ e $\beta=\pi / 2$, foi possível testar a validade do modelo. A Figura 7 compara as energias ab initio com as obtidas pelo modelo para a série do gás nobre. Existem diferenças na região do poço de potencial, porém a concordância é muito boa, principalmente para os gases de baixo peso molecular, como é o caso do hélio e neônio. A Figura 8 ilustra os termos da expansão para o caso do $\mathrm{H}_{2} \mathrm{O}_{2} \cdots \mathrm{Ar}$, onde são mostrados os potenciais médios de cada configuração, $\mathrm{v}_{00}{ }^{0}(\mathrm{C} ; \mathrm{r}), \mathrm{v}_{00}{ }^{0}(\mathrm{E} ; \mathrm{r}), \mathrm{v}_{00}{ }^{0}(\mathrm{~T} ; \mathrm{r})$, e as componentes isotrópica, $\overline{\mathrm{v}}_{00}{ }^{0}(r)$, e anisotrópicas, $\overline{\mathrm{v}}_{01}{ }^{1}(r)$ e $\overline{\mathrm{v}}_{02}{ }^{2}(r)$, de acordo com as Equações (14), sendo que o termo isotrópico pode ser medido experimentalmente e comparado com outros sistemas similares, como é o caso do $\mathrm{H}_{2} \mathrm{~S}_{2}-\mathrm{Rg}, \mathrm{H}_{2} \mathrm{O}-\mathrm{Rg}$ e $\mathrm{H}_{2} \mathrm{~S}-\mathrm{Rg}$.

\section{Sistema $\mathrm{H}_{2} \mathrm{~S}_{2} \cdots \mathbf{R g}$}

Foram calculadas 81 energias ab initio para as onze configurações principais, conforme Tabela 2, para o $\mathrm{H}_{2} \mathrm{~S}_{2}$ .. Rg, com $\mathrm{Rg}=\mathrm{He}-\mathrm{Kr}$. Todas as energias foram obtidas em função da distância $r$ (Figura 1b) entre o gás nobre e o centro de massa da molécula de $\mathrm{H}_{2} \mathrm{~S}_{2}$.

As Figuras de 9 - 12 comparam as energias ab initio com as obtidas via o modelo proposto para o $\mathrm{He}, \mathrm{Ne}$, Ar e Kr, respectivamente. Como apresentado no caso dos sistemas $\mathrm{H}_{2} \mathrm{O}_{2} \cdots \mathrm{Rg}$, as energias estão agrupadas em três regiões: a de equilíbrio, inferior, a trans, intermediária, e a cis, a superior, comum para todos os sistemas analisados, porém no caso do $\mathrm{H}_{2} \mathrm{~S}_{2}$ a barreira trans é superior a do $\mathrm{H}_{2} \mathrm{O}_{2}$.

Outra tendência comum e que também foi observada no caso do $\mathrm{H}_{2} \mathrm{O}_{2} \cdots \mathrm{Rg}$, é que a distância do mínimo e a

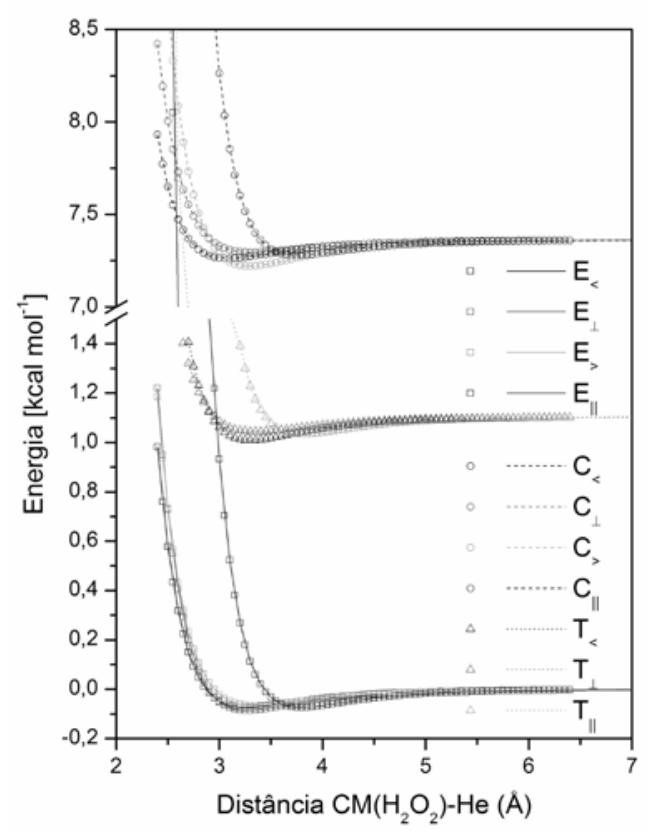

Figura 2: Energia de interação em função da distância $\mathrm{He}$ - centro de massa da molécula de $\mathrm{H}_{2} \mathrm{O}_{2}$ nas onze configurações principais (símbolos são os pontos ab initio e as linhas os potenciais oriundos do modelo desenvolvido).

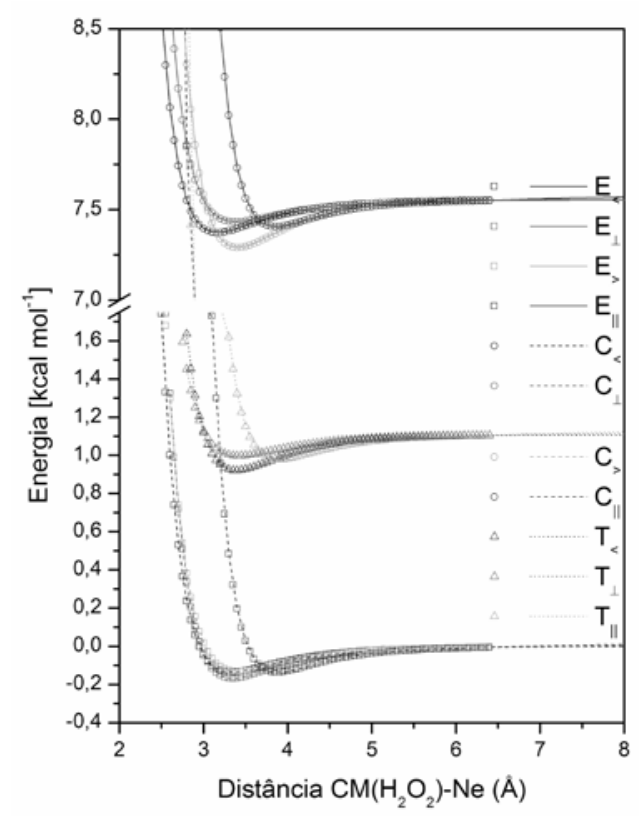

Figura 3: Energia de interação em função da distância $\mathrm{Ne}$ - centro de massa da molécula de $\mathrm{H}_{2} \mathrm{O}_{2}$ nas onze configurações principais (símbolos são os pontos ab initio e as linhas os potenciais oriundos do modelo desenvolvido). 


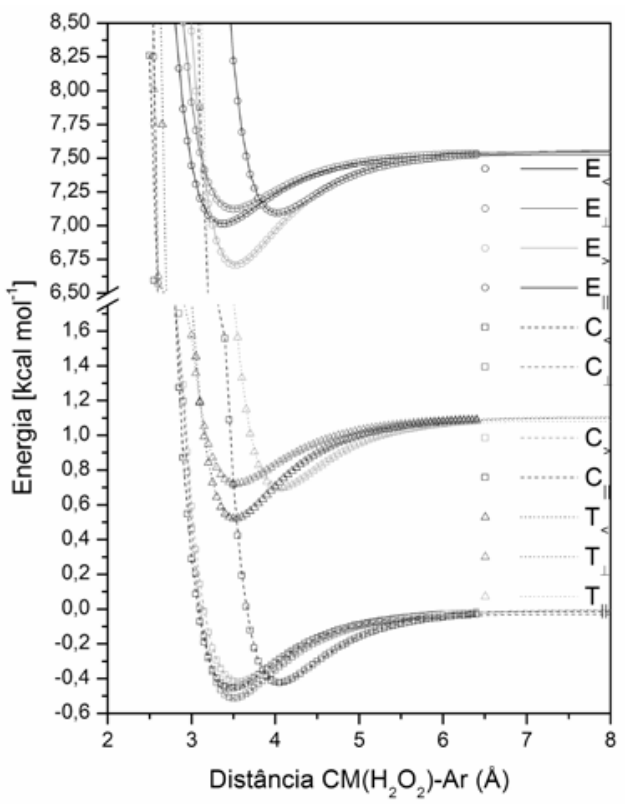

Figura 4: Energia de interação em função da distância $\mathrm{Ar}$ - centro de massa da molécula de $\mathrm{H}_{2} \mathrm{O}_{2}$ nas onze configurações principais (símbolos são os pontos ab initio e as linhas os potenciais oriundos do modelo desenvolvido).

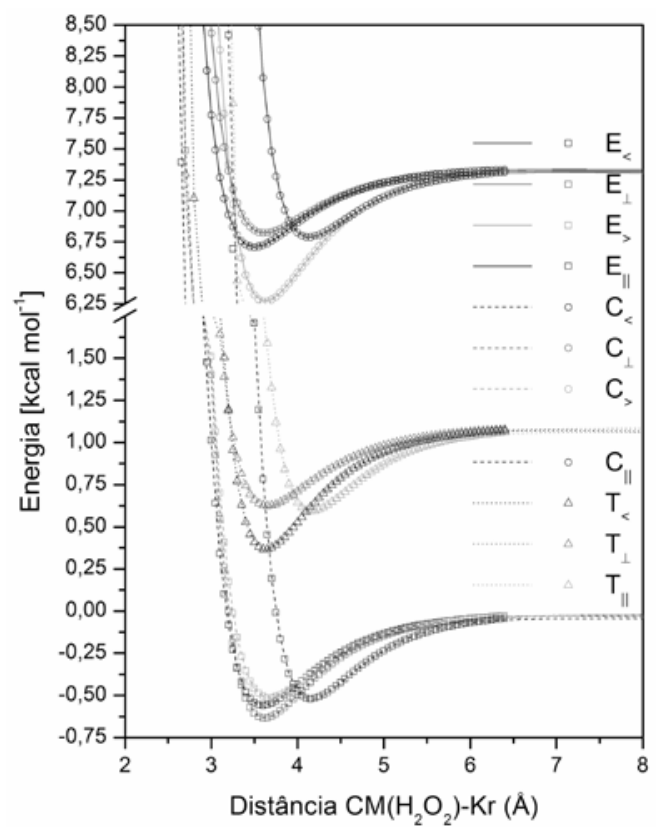

Figura 5: Energia de interação em função da distância $\mathrm{Kr}$ - centro de massa da molécula de $\mathrm{H}_{2} \mathrm{O}_{2}$ nas onze configurações principais (símbolos são os pontos ab initio e as linhas os potenciais oriundos do modelo desenvolvido).

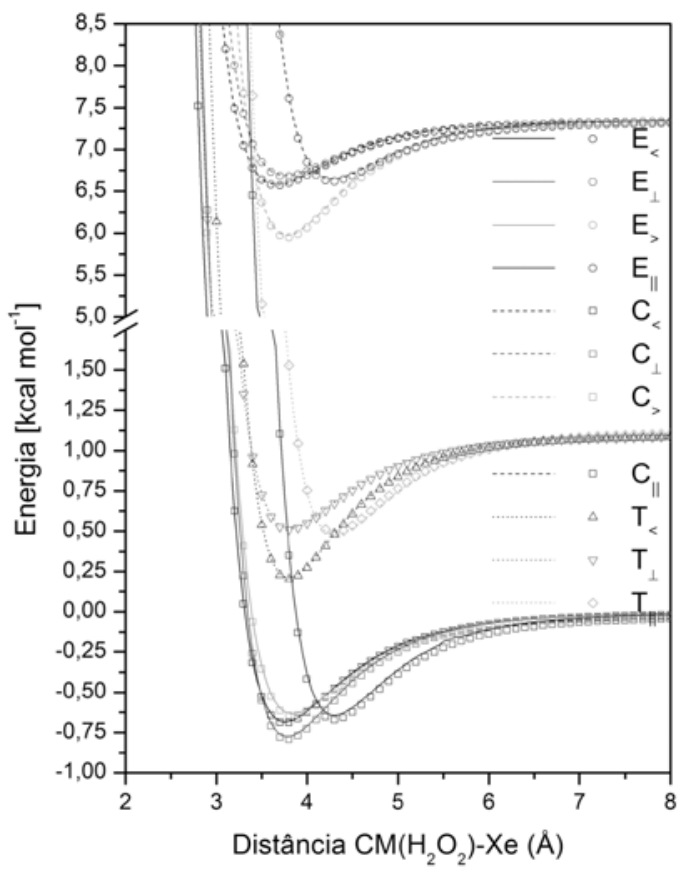

Figura 6: Energia de interação em função da distância Xe - centro de massa da molécula de $\mathrm{H}_{2} \mathrm{O}_{2}$ nas onze configurações principais (símbolos são os pontos ab initio e as linhas os potenciais oriundos do modelo desenvolvido).

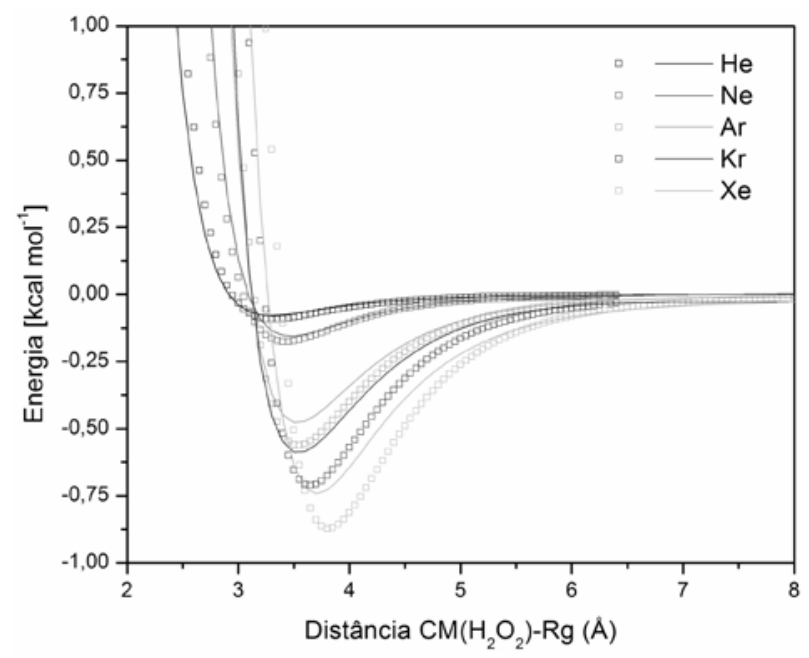

Figura 7: Energia de interação em função da distância entre o gás nobre e o centro de massa da molécula de $\mathrm{H}_{2} \mathrm{O}_{2}$, para a geometria de equilíbrio $\left(\gamma=112.54^{\circ}\right)$, considerando o gás nobre se aproximando na direção $\alpha=\gamma_{\mathrm{eq}} / 2$ e $\beta=\pi / 2$, (símbolos são os pontos ab initio e as linhas os potenciais oriundos do modelo desenvolvido). 




Figura 8: Dependência da distância átomo-molécula para as representações isotrópicas (linhas contínuas) e anisotrópicas (linhas tracejadas) dos momentos da expansão em harmônicos hiperesféricos do $\mathrm{H}_{2} \mathrm{O}_{2}$-Ar.



Figura 9: Energia de interação em função da distância $\mathrm{He}$ - centro de massa da molécula de $\mathrm{H}_{2} \mathrm{X}_{2}$ nas onze configurações principais (símbolos são os pontos ab initio e as linhas os potenciais oriundos do modelo desenvolvido).

energia de interação aumentam quando se parte de um átomo leve, He, para um átomo pesado, $\mathrm{Kr}$.

Utilizando uma configuração teste, para a geometria

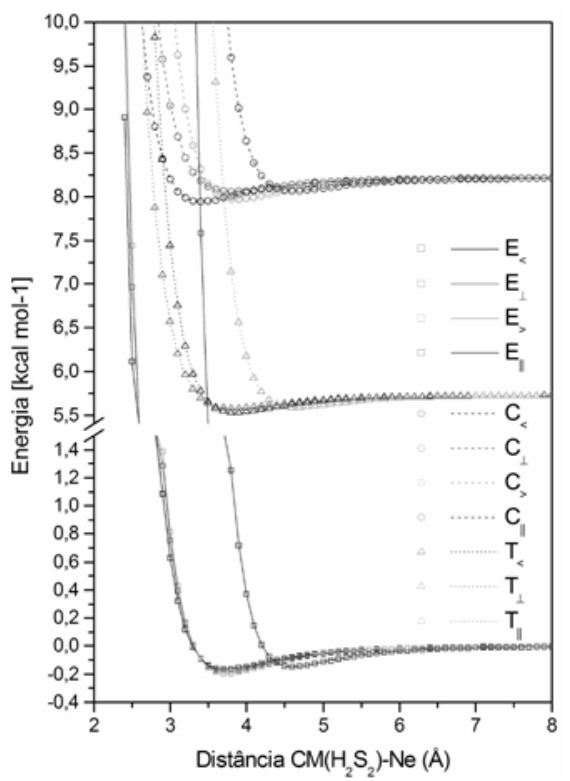

Figura 10: Energia de interação em função da distância $\mathrm{Ne}$ - centro de massa da molécula de $\mathrm{H}_{2} \mathrm{X}_{2}$ nas onze configurações principais (símbolos são os pontos ab initio e as linhas os potenciais oriundos do modelo desenvolvido).

de equilíbrio $\left(\gamma=91.05^{\circ}\right)$ com $\alpha=\gamma$ eq $/ 2$ e $\beta=\pi / 2$, foi possível testar a validade do modelo. A Figura 13 compara as energias ab initio com as obtidas pelo modelo para a série do gás nobre. Existem diferenças na região do poço de potencial, porém a concordância é muito boa, principalmente para os gases de baixo peso molecular, como é o caso do hélio e neônio.

A Figura 14 ilustra os termos da expansão para o caso do $\mathrm{H}_{2} \mathrm{~S}_{2} \cdots$ Ar, onde são mostrados os potenciais médios de cada configuração, $\overline{\mathrm{v}}_{00}{ }^{0}(\mathrm{C} ; r), \overline{\mathrm{v}}_{00}{ }^{0}(\mathrm{E} ; r), \overline{\mathrm{v}}_{00}{ }^{0}(\mathrm{~T} ; r)$, e as componentes isotrópica, $\overline{\mathrm{v}}_{00}{ }^{0}(r)$, e anisotrópicas, $\overline{\mathrm{v}}_{01}{ }^{1}(r)$ e $\overline{\mathrm{v}}_{02}{ }^{2}(r)$, de acordo com as equações (14), sendo que o termo isotrópico pode ser medido experimentalmente e comparado com outros sistemas similares, como é o caso do $\mathrm{H}_{2} \mathrm{~S}_{2} \cdots \mathrm{Rg}, \mathrm{H}_{2} \mathrm{O} \cdots \mathrm{Rg}$ e $\mathrm{H}_{2} \mathrm{~S} \cdots \mathrm{Rg}$.

\section{CORRELAÇÃO}

A partir do termo isotrópico, $\overline{\mathrm{V}}_{00}{ }^{0}(r)$, é possível obter a distância de equilíbrio do sistema, $\mathrm{H}_{2} \mathrm{X}_{2} \cdots \mathrm{Rg}$, e a energia de interação. A figura 15 compara estes parâmetros com sistemas semelhantes, $\mathrm{H}_{2} \mathrm{O} \cdots \mathrm{Rg}$ e $\mathrm{H}_{2} \mathrm{~S} \cdots \mathrm{Rg}$. Em todos os casos a energia e distância aumentam com o aumento 


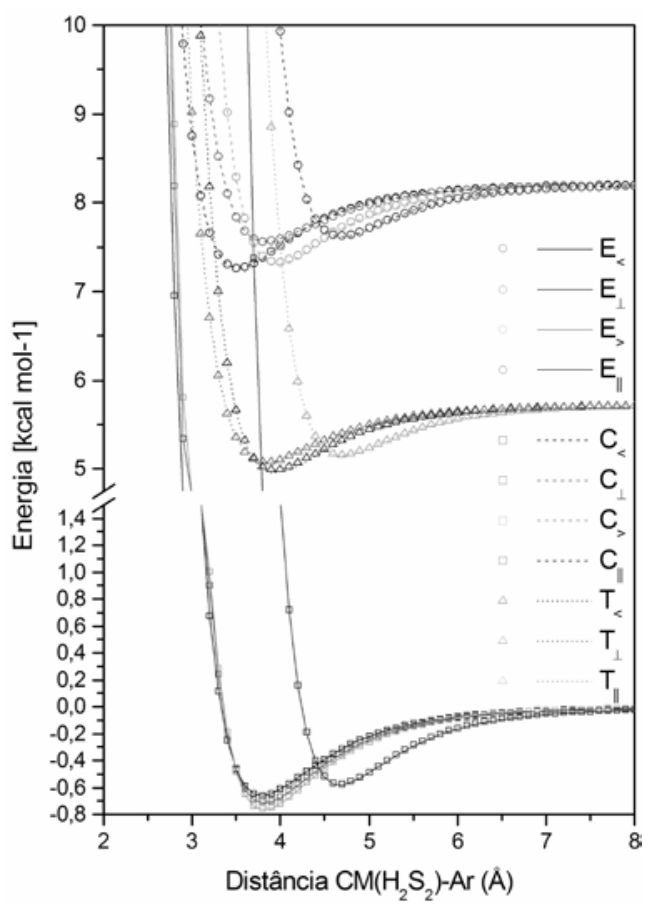

Figura 11: Energia de interação em função da distância $\mathrm{Ar}$ - centro de massa da molécula de $\mathrm{H}_{2} \mathrm{X}_{2}$ nas onze configurações principais (símbolos são os pontos ab initio e as linhas os potenciais oriundos do modelo desenvolvido).

do peso molecular do gás nobre.

Duas conclusões importantes são obtidas a partir destas figuras, as energias e distâncias aumentam para todos os sistemas, indo do He para o Xe e para um mesmo gás nobre a energia aumenta na seqüência $\mathrm{H}_{2} \mathrm{O}$ $\cdots \mathrm{Rg}<\mathrm{H}_{2} \mathrm{~S} \cdots \mathrm{Rg}<\mathrm{H}_{2} \mathrm{O}_{2} \cdots \mathrm{Rg}<\mathrm{H}_{2} \mathrm{~S}_{2} \cdots \mathrm{Rg}$, ao passo que a distância aumenta na seqüência $\mathrm{H}_{2} \mathrm{O} \cdots \mathrm{Rg}<\mathrm{H}_{2} \mathrm{O}_{2}$ $\cdots \mathrm{Rg}<\mathrm{H}_{2} \mathrm{~S} \cdots \mathrm{Rg}<\mathrm{H}_{2} \mathrm{~S}_{2} \cdots \mathrm{Rg}$. Este comportamento está de acordo com o que é esperado para forças de van der Waals e as correspondentes polarizabilidades atômicas e moleculares das espécies envolvidas nestes sistemas 33,34 .

\section{Conclusão}

Foi provado que a química quântica é uma ferramenta poderosa para resolver vários problemas, como: otimização de geometria, determinação de momento de dipolo, altura de barreiras para mudança de quiralidade, cálculo de energia de interação, etc., o que são cruciais para o estudo da dinâmica molecular e de vários

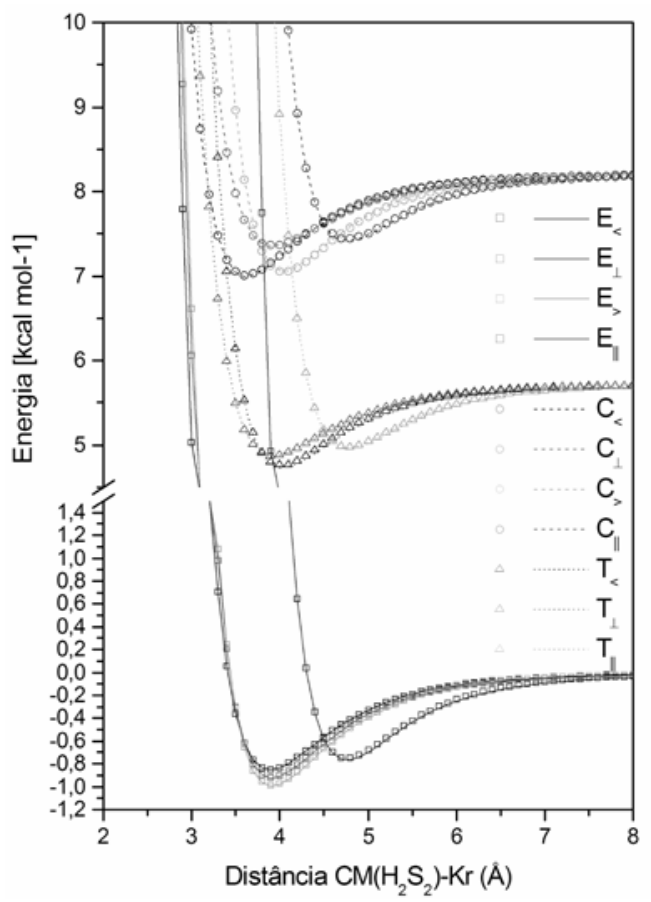

Figura 12: Energia de interação em função da distância $\mathrm{Kr}$ - centro de massa da molécula de $\mathrm{H}_{2} \mathrm{X}_{2}$ nas onze configurações principais (símbolos são os pontos ab initio e as linhas os potenciais oriundos do modelo desenvolvido).

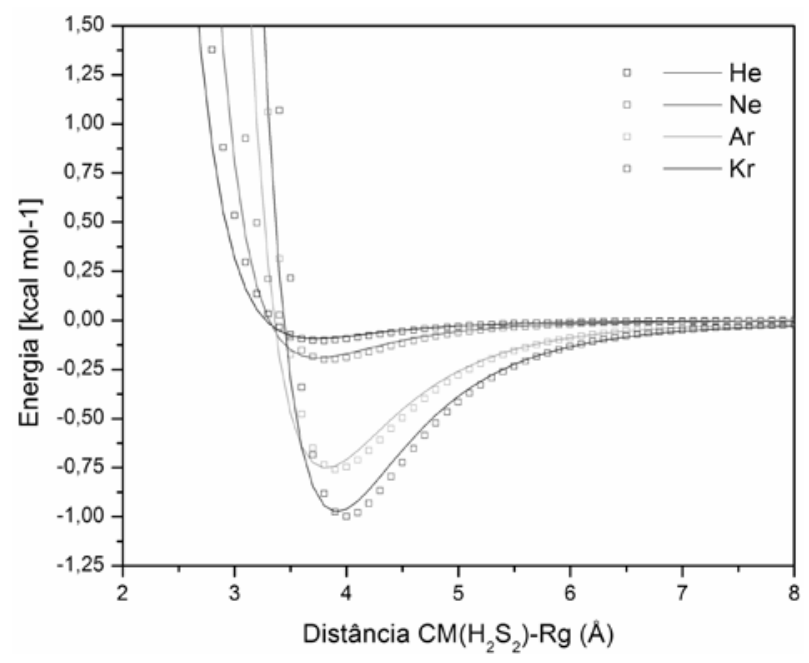

Figura 13: Energia de interação em função da distância entre o gás nobre e o centro de massa da molécula de $\mathrm{H}_{2} \mathrm{~S}_{2}$, para a geometria de equilíbrio $\left(\gamma=91.05^{\circ}\right)$, considerando o gás nobre se aproximando na direção $\alpha=\gamma_{\mathrm{eq}} / 2$ e $\beta=\pi / 2$, (símbolos são os pontos ab initio e as linhas os potenciais oriundos do modelo desenvolvido). 


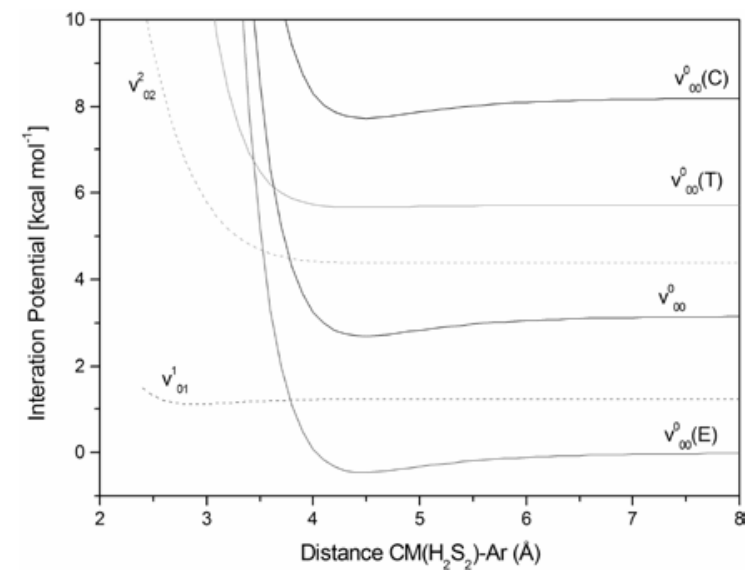

Figura 14: Dependência da distância átomo-molécula para as representações isotrópicas (linhas contínuas) e anisotrópicas (linhas tracejadas) dos momentos da expansão em harmônicos hiperesféricos do $\mathrm{H}_{2} \mathrm{~S}_{2}$-Ar.

fenômenos presentes na atmosfera.

Neste contexto, foi proposta uma SEP escrita em termos dos harmônicos hiperesféricos para descrever os sistemas $\mathrm{H}_{2} \mathrm{X}_{2} \cdots \mathrm{Rg}$ e de acordo com esses dados apresentados, foi possivel reproduzir com excelente concordancia os dados ab initio para os gases nobres e diferentes moléculas quirais empregados. Um dos resultados mais importantes desta expansão são os termos isotrópicos e anisotrópicos do potencial em função da distância, que podem ser medidos experimentalmente e comparados com outros sistemas similares. Comparando os sistemas $\mathrm{H}_{2} \mathrm{O} \cdots \mathrm{Rg}, \mathrm{H}_{2} \mathrm{~S} \cdots \mathrm{Rg}, \mathrm{H}_{2} \mathrm{O}_{2} \cdots \mathrm{Rg}$ e $\mathrm{H}_{2} \mathrm{~S}_{2} \cdots$ $\mathrm{Rg}$ verificou-se que as energias e distâncias aumentam com o aumento do peso molecular do gás nobre, e que para um mesmo $\mathrm{Rg}$ a energia é na seqüência $\mathrm{H}_{2} \mathrm{O} \cdots \mathrm{Rg}$ $<\mathrm{H}_{2} \mathrm{~S} \cdots \mathrm{Rg}<\mathrm{H}_{2} \mathrm{O}_{2} \cdots \mathrm{Rg}<\mathrm{H}_{2} \mathrm{~S}_{2} \cdots \mathrm{Rg}$, ao passo que a distância aumenta na seqüência $\mathrm{H}_{2} \mathrm{O} \cdots \mathrm{Rg}<\mathrm{H}_{2} \mathrm{O}_{2} \cdots \mathrm{Rg}$ $<\mathrm{H}_{2} \mathrm{~S} \cdots \mathrm{Rg}$.

\section{Agradecimentos}

Os autores agradecem ao FAPESP, à PrP/UEG pelo apoio financeiro, ao Prof. Vincenzo Aquilanti, da Università di Perúgia, Perúgia - Italy, pelas discussões que contribuíram para o enriquecimento deste trabalho.

\section{REFERÊNCIAS}

1. V. Aquilanti, D. Ascenzi, M. Bartolomei, D.
Cappelletti, S. Cavalli, M. De Castro Vitores, and F. Pirani. Phys. Rev. Lett., 82:69, 1999.

2. V. Aquilanti, D. Ascenzi, M. Bartolomei, D. Cappelletti, S. Cavalli, M. De Castro Vitores, and F. Pirani. J. Am. Chem. Soc., 121:10794, 1999.

3. V. Aquilanti, M. Bartolomei, D. Cappelletti, E. Carmona-Novillo, , and F. Pirani. J. Chem. Phys., 117:615, 2002.

4. V. Aquilanti, M. Bartolomei, D. Cappelletti, E. Carmona-Novillo, , and F. Pirani. Phys. Chem. Chem. Phys., 3:3891, 2001.

5. E. Carmona-Novillo, F. Pirani, and V. Aquilanti. Int. J. Quantum Chem., 99:616, 2004.

6. A. F. A. Vilela, P. R. P. Barreto, R. Gargano, and C. R. Cunha. Chem. Phys. Lett., 427:29, 2006.

7. V. Aquilanti, D. Ascenzi, M. Bartolomei, D. Cappelletti, S. Cavalli, M. De Castro Vitores, and F. Pirani. Phys. Rev. Lett., 82:69, 1999.

8. V. Aquilanti, D. Ascenzi, M. Bartolomei, D. Cappelletti, S. Cavalli, M. De Castro Vitores, and F. Pirani. J. Am. Chem. Soc., 121:10794, 1999.

9. V. Aquilanti, M. Bartolomei, D. Cappelletti, E. Carmona-Novillo, , and F. Pirani. J. Chem. Phys., 117:615, 2002.

10. V. Aquilanti, M. Bartolomei, D. Cappelletti, E. Carmona-Novillo, , and F. Pirani. Phys. Chem. Chem. Phys., 3:3891, 2001.

11. E. Carmona-Novillo, F. Pirani, and V. Aquilanti. Int. J. Quantum Chem., 99:616, 2004.

12. A. F. A. Vilela, P. R. P. Barreto, R. Gargano, and C. R. Cunha. Chem. Phys. Lett., 427:29, 2006.

13. A. F. A. Vilela, P. R. P. Barreto, R. Gargano, and C. R. Cunha. Chem. Phys. Lett., (em preparação).

14. V. Aquilanti, E. Cornicchi, M. M. Teixidor, N. Saendig, F. Pirani, and D. Cappelletti. Angew. Chem., Int. Ed. 44:2356, 2005.

15. D. Cappelletti, P.R.P. Barreto, A.F.A. Vilela, R. Gargano, F. Pirani, and V. Aquilanti. J. Chem. Phys., 125:133111, 2006. 
16. V. Aquilanti, D. Cappelletti, F. Pirani, and L. F. Roncaratti. Int. J. Mass Spectrom., 2008 (in press).

17. G.S. Maciel, A.C. Bitencourt, M. Ragni, and V. Aquilanti. Chem. Phys. Lett., 432:383, 2006.

18. G.S. Maciel, P.R.P. Barreto, F. Palazzetti, A. Lombardi and V. Aquilanti. J. Chem. Phys., 129, 164302, 2008.

19. P.R.P. Barreto, A.F.A. Vilela, A. Lombardi, G.S. Maciel, F. Palazzetti, and V. Aquilanti. J. Phys. Chem. A, 111, 12754-12762, 2007.

20. G.S. Maciel, A.C. Bitencourt, M. Ragni, and V. Aquilanti. Int. J. Quantum Chem., 107:2697-2707, 2007.

21. G.S. Maciel, A.C. Bitencourt, M. Ragni, and V. Aquilanti. J. Phys. Chem. A, 111, 12604-12610, 2007.

22. S. François, I. Sowka, A. Monod, B. TemimeRoussel, J.M. Laugier, and H. Wortham. Atmospheric Research, 74:525-545, 2005.

23. V. Vaida. Int. J. Photoenergy, 7:61-70, 2005.

24. M. Lee, Brian G. Heikes, and Daniel W. O’Sullivan. Atmospheric Environment, 34:3475-3494, 2000.

25. M. C. Daza, J. A. Dobado, J. M. Molina, P. Salvador, M. Duran, and J. L. Villaveces. J. Chem. Phys., 110:11806-11813, 1999.

26. M. C. Daza, J. A. Dobado, J. M. Molina, and J. L. Villaveces. Phys. Chem. Chem. Phys., 2:4087-4097, 2000.

27. J. M. Molina, J. A. Dobado, M. C. Daza, and J. L. Villaveces. J. Mol. Struct. (Theochem), 580:117-126, 2002.

28. M. Elange, R. Parthasarathi, V. Subramanian, C. N. Ramachandran, and N. J. Sathyamurthy. J. Phys. Chem. A, 110:6294-6300, 2006.

29. D. A. Varshalovich, A. N. Moskalev, and V. K. Khersonskii. Quantum Theory of Angular Momentum. World Scientific, Singapore, 1988.

30. Frisch, M. J.; Trucks, G. W.; Schlegel, H. B.; Scuseria, G. E.; Robb, M. A.; Cheeseman, J. R.; Montgomery, J. A., Jr.; Vreven, T.; Kudin, K. N.; Burant, J. C.; Millam,
J. M.; Iyengar, S. S.; Tomasi, J.; Barone, V.; Mennucci, B.; Cossi, M.; Scalmani, G.; Rega, N.; Petersson, G. A.; Nakatsuji, H.; Hada, M.; Ehara, M.; Toyota, K.; Fukuda, R.; Hasegawa, J.; Ishida, M.; Nakajima, T.; Honda, Y.; Kitao, O.; Nakai, H.; Klene, M.; Li, X.; Knox, J. E.; Hratchian, H. P.; Cross, J. B.; Bakken, V; Adamo, C.; Jaramillo, J.; Gomperts, R.; Stratmann, R. E.; Yazyev, O.; Austin, A. J.; Cammi, R.; Pomelli, C.; Ochterski, J. W.; Ayala, P. Y.; Morokuma, K.; Voth, G. A.; Salvador, P.; Dannenberg, J. J.; Zakrzewski, V. G.; Dapprich, S.; Daniels, A. D.; Strain, M. C.; Farkas, O.; Malick, D. K.; Rabuck, A. D.; Raghavachari, K.; Foresman, J. B.; Ortiz, J. V.; Cui, Q.; Baboul, A. G.; Clifford, S.; Cioslowski, J.; Stefanov, B. B.; Liu, G.; Liashenko, A.; Piskorz, P.; Komaromi, I; Martin, R. L.; Fox, D. J.; Keith, T.; Al-Laham, M. A.; Peng, C. Y.; Nanayakkara, A.; Challacombe, M.; Gill, P. M. W.; Johnson, B.; Chen, W.; Wong, M. W.; Gonzalez, C.; Pople J. A. Gaussian 03, revision C.02; Technical report; Gaussian, Inc.: Wallingford, CT, 2004.

31. K.A. Peterson, D. Figgen, E. Goll, H. Stoll, , and M. Dolg. J. Chem. Phys., 119(21):11113-11123, 2003.

32. Basis sets were obtained from the Extensible Computational Chemistry Environment Basis Set Database, Version 02/02/06, as developed and distributed by the Molecular Science Computing Facility, Environmental and Molecular Sciences Laboratory which is part of the Pacific Northwest Laboratory, P.O. Box 999, Richland, Washington 99352, USA, and funded by the U.S. Department of Energy. The Pacific Northwest Laboratory is a multiprogram laboratory operated by Battelle Memorial Institute for the U.S. Department of Energy under contract DE-AC06-76RLO 1830.

33. S. F. Boys and F. Bernardi. Mol. Phys., 19(55), 1970.

34. R. L. Redington, W. B. Olson, and P. C. Cross. J. Chem. Phys., 36:1311-1326, 1962.

35. Jacek Koput. Chemical Physics Letters, 236:516-520, 1995.

36. R.H. Hunt, R. A. Leacock, C. W. Peters, and K. T. Hecht. J. Chem. Phys., 42:1931-1946, 1965.

37. C. J. Marsden and B. J. Smith. J. Phys. Chem., 92: 


\section{Artigo 1}

347-353, 1988.

38. D.A. Dixon and D. Zeroka and J. J. Wendeloski and Z. R. Wasserman. J. Phys. Chem., 89: 5334-5336, 1985.

39. F. Pirani, G. S. Maciel, D. Cappelletti, and V. Aquilanti. Int. Rev. Phys. Chem., 25(1-2):165, 199, 2006.

40. P.R.P. Barreto, F. Palazzetti, G.S. Maciel, A.F.A. Vilela, A. Lombardi, Phys. Scripta (em preparação).

\section{Patrícia R. P. Barreto*1, Alessandra F. Albernaz ${ }^{2}$, Glauciete S. Maciel ${ }^{3}$, Federico Palazzetti ${ }^{3}$, Andrea Lombardi ${ }^{3}$ \& Gaia Grossi}

${ }^{1}$ Laboratório Associado de Plasma, Instituto Nacional de Pesquisas Espaciais, CEP: 12247-970, São José dos Campos, São Paulo, Brasil.

${ }^{2}$ Departamento de Engenharia e Física, Universidade Federal de Rondônia, CEP: 78000-900, Porto Velho, RO, Brasil

${ }^{3}$ Dipartimento di Chimica, Università di Perugia, 06123 Perugia, Itália.

*E-mail: patricia@plasma.inpe.br 